\title{
Linoleic acid positioning in psi factor producing oxygenase A, a fusion protein with an atypical cytochrome P450 activity
}

\author{
Christian Koch $^{1}$, Alistair J. Fielding ${ }^{2}$, Florian Brodhun ${ }^{1}$, Marina Bennati ${ }^{2}$ and Ivo Feussner ${ }^{1}$ \\ 1 Department of Plant Biochemistry, Albrecht-von-Haller-Institute for Plant Sciences, Georg-August-University, Göttingen, Germany \\ 2 Max-Planck Institute for Biophysical Chemistry, Göttingen, Germany
}

\author{
Keywords \\ Aspergillus nidulans; bifunctional enzyme: \\ enzyme mechanism; fatty acid dioxygenase; \\ oxylipin formation

\section{Correspondence} \\ Ivo Feussner, Department of Plant \\ Biochemistry, Albrecht-von-Haller-Institute \\ for Plant Sciences, Georg-August-University, \\ Justus-von-Liebig-Weg 11, \\ D-37077 Göttingen, Germany \\ Fax: +49551395749 \\ Tel: +49551395743 \\ E-mail: ifeussn@uni-goettingen.de
}

(Received 24 June 2011, revised 2 September 2011, accepted 13 September 2011)

doi:10.1111/j.1742-4658.2011.08352.x
Psi factor producing oxygenases (Ppos) are fusion proteins consisting of a peroxidase-like functionality in the $\mathrm{N}$-terminus and a $\mathrm{P} 450$-fold in the C-terminal part of the polypeptide chain. It was shown that they are responsible for the production of oxidized fatty acids that play a pivotal role in the control of fungal colonization of plant and mammalian hosts. The similarity of the primary structure of the single domains to various host-derived oxylipin-forming enzymes and functional conservation of these enzymatic activities was the basis for prediction of the $3 \mathrm{D}$ conformations of the single domains of a prototype Ppo enzyme. We were able to predict a putative substrate binding pocket in the $\mathrm{N}$-terminal domain of the enzyme and support this finding by site-directed mutagenesis. With the proposed substrate binding mode all known determinants of oxygen insertion are in a reasonable spatial arrangement for catalysis. Additionally, we could identify an arginine and show its involvement in substrate binding by kinetic analysis of the respective variant. While substrate position in the dioxygenase domain is well defined, our results indicate that the substrate binding to the P450 domain is rather unconstrained. Nevertheless an asparagine residue within the I-helix is shown to be involved in catalysis and promotes a shortcut of the typical P450 reaction cycle. Taken together, the results presented here exemplify that fatty acids are oxidized in all kingdoms of life by structural and functional highly conserved enzymes.

\section{Introduction}

Oxidized fatty acids and their metabolites, termed oxylipins, act as signaling molecules in virtually all kingdoms of life [1]. For the model ascomycete Aspergillus nidulans, it has been shown in recent years that specific oxylipins, known as precocious sexual inducers (psi factors) play a crucial role for the balance of different developmental processes and the formation of mycotoxins [2-4]. From a chemical point of view, these signaling molecules are fatty acids with 18 carbon atoms, which are hydroxylated at distinct positions. The finding that these oxylipins are involved in processes related to fungal pathogenicity and that there is a chemical similarity between the pathogen-derived signal molecules and host-derived oxylipins led to the proposal that they might play a central role in pathogen-host interaction and trans-species communication, the so-called cross-kingdom signaling [5-7]. After the genome of $A$. nidulans was sequenced, three genes

\footnotetext{
Abbreviations

AOS, allene oxide synthase; DiHODE, dihydroxy octadecadienoic acid; DOX, dioxygenase; HODE, hydroxy octadecadienoic acid; HPODE, hydroperoxy octadecadienoic acid; LA, octadecadienoic acid; LDS, linoleate diol synthase; P450, cytochrome P450; PGHS, prostaglandin $\mathrm{H}_{2}$ synthase; PGIS, prostacyclin synthase; Ppo, psi factor producing oxygenase; psi factor, precocious sexual inducer.
} 
putatively involved in formation of these important signaling molecules have been identified and were named pро $A$, рроB and $р$ роC [8]. With the advent of more and more fungal genomes sequenced, it became obvious that the enzymes encoded by these and homologue genes are conserved in virtually all ascomycetes $[1,8]$ and play a pivotal role in conferring virulence of ascomycetes towards mammalian and plant hosts $[3,7,9]$. These psi factor producing oxygenases (Ppos) are heme enzymes consisting of two distinct enzymatic functionalities [10]. To date the enzyme encoded by A. nidulans pро $A$, beside the 7,8-linoleate diol synthase (7,8-LDS) from Gaeumannomyces graminis [11], is by far the biochemically best characterized [10,12-14]. It was shown that PpoA is a bifunctional, tetrameric heme enzyme with a molecular mass of $\sim 480 \mathrm{kDa}$ [10,12-14]. Both domains show functional similarities and sequence homologies to known oxylipin-forming enzymes from mammals and plants. In a first step the substrate, an unsaturated fatty acid with 18 carbons, is converted to a C8-hydroperoxy fatty acid at the $\mathrm{N}$-terminal enzyme domain that is proposed to have high structural and functional similarity to the mammalian prostaglandin $\mathrm{H}_{2}$ synthase (PGHS). The C-terminal domain of the polypeptide chain is predicted to possess a conserved P450-fold and catalyzes the rearrangement of the N-terminally formed hydroperoxy fatty acid to a dihydroxy fatty acid. Based on the reaction catalyzed, we propose that the $\mathrm{P} 450$ domain of the fungal PpoA enzyme shares the highest similarity to the subfamily of class III P450s [15]. This subclass comprises all $\mathrm{P} 450$ s that do not require external electron donors and thus do not catalyze typical P450 monooxygenations, e.g. they do not insert oxygen, derived from molecular oxygen. The enzymes of this class instead utilize fatty acid peroxides as substrates for rearrangement reactions to yield diverse products. Interestingly, all known enzymes of this subclass, e.g. Cyp5 (thromboxane A synthase), Cyp8a (prostacyclin synthase, PGIS) and Cyp74 [allene oxide synthase (AOS), divinyl ether synthase and hydroperoxide lyase], are involved in the biosynthesis of oxylipins in various species [16].

While information on the mechanism of the two reaction steps, fatty acid dioxygenation and rearrangement of the formed hydroperoxy fatty acid, has accumulated through the last few years [10,12-14], structural information on the enzyme is still scarce. Therefore, in this work we predict the 3D fold of the single PpoA domains, independently. Based on these hypothetical models, we could validate the proposed conservation of oxylipin-forming enzymes and derive new determinants for fatty acid oxidation in the single domains of the prototype enzyme PpoA.

\section{Results}

\section{Predicted substrate binding sites}

The structure of both PpoA apodomains was predicted using the I-TASSER algorithm as outlined in Materials and methods. For evaluation of the model, we compared the predicted structures with crystal structures of prototype enzymes of the respective enzyme families (Fig. 1A,B). The core region of the dioxygenase (DOX) domain structurally aligned with the 309 a-carbons of the solved structure of mouse PGHS2 (PDB 3HS5) to an rmsd of $0.95 \AA$, while there was a sequence identity of $20 \%$ between the two enzymes. The P450 domain of PpoA aligned to an rmsd of $1.17 \AA$ over $113 \mathrm{C} \alpha$-atoms with guayule AOS (Cyp74A2; PDB 3DBM; 17\% amino acid identity of the sequences). In contrast to this result, the rmsd to PGIS (Cyp8A; PDB 3B99), one of the mammalian members of class III $\mathrm{P} 450 \mathrm{~s}$, was higher. For the purpose of validating both the model and the proposed homology, substrate was positioned in the domains according to its orientation in the homologue enzymes (Fig. 1C-F).

\section{DOX domain}

In mPGHS a positively charged arginine residue is involved in binding of the substrate. Within the crystal structure, the guanidinium group of this residue (Arg120 in mPGHS2) is $4.6 \AA$ apart from the carboxy group of the substrate. In the in silico predicted DOX domain of PpoA the residue Arg336 is within this radius and the corresponding ionic bond length would be $4.5 \AA$ (Fig. 1E). The vicinity of the potential substrate entrance harbors some additional positively charged amino acids: Arg329, Arg555, Lys80, Lys106 and Lys325. Nevertheless, within the modeled structure these groups seem to be too far apart to participate in ionic interactions with the substrate ( $>10 \AA$ ). In addition a number of amino acids that have been previously reported to play a role in the catalysis of PpoA and 7,8-LDS were confirmed by our model. Most important, Tyr374 (PpoA numbering) was proposed to be involved in the radical chemistry of hydrogen abstraction from $\mathrm{C} 8$ of the fatty acid substrate. The importance of this amino acid was later confirmed with the purified enzyme [10], and EPR spectroscopy proved that this residue is indeed involved in radical chemistry [13]. Within the modeled domain, this residue is in a 

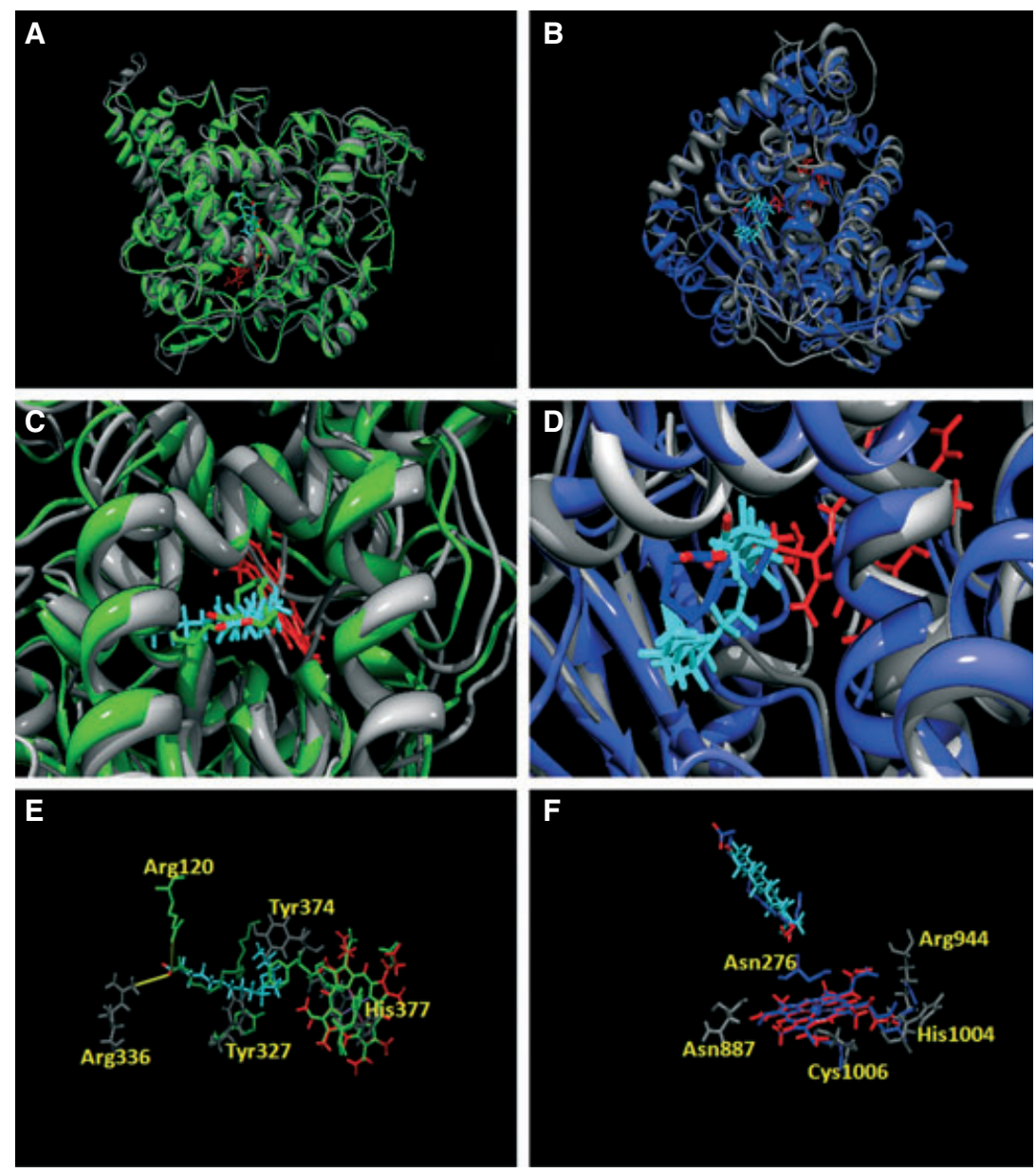

Fig. 1. (A) Proposed structure of the PpoA DOX domain. Due to functional and sequence similarities, the mammalian oxylipin-forming enzyme PGHS can serve as a scaffold for homology modeling of the DOX domain. The predicted structure of this N-terminal domain was superimposed with the structure of mouse PGHS-2 (PDB 3HS5; green ribbon; heme cofactor depicted in red). A possible orientation of LA (cyan) in the hypothetical model of the DOX domain is shown. (B) Proposed structure of the PpoA P450 domain. The amino acid sequence and the reaction catalyzed classify this domain as a class III P450. Shown is the superimposition of the predicted domain with the crystal structure of Parthenium argentatum AOS, one member of class III P450s (PDB 3DBM; blue ribbon; heme cofactor depicted in red). A possible orientation of 8-HPODE (cyan) in the hypothetical model of the P450 domain is shown. (C) Zoom into the substrate binding pocket of the DOX domain. (D) Zoom into the substrate binding pocket of the P450 domain. (E) Residues crucial for DOX activity. The proximal side of the heme is shown. The known distal and proximal histidines of the heme are conserved between the hypothetical PpoA model and PGHS-2. Besides hydrophobic interactions, ionic interaction of the fatty acid's carboxy group with Arg336 is of importance for formation of enzymesubstrate complex. In mPGHS-2 Arg120 was proposed to fulfill this role. Although its position relative to the fatty acid's carboxy group is not conserved, the length of the putative salt bridge is maintained. Tyr374 is supposed to be involved in the radical mechanism, leading to abstraction of hydrogen from C8 of the fatty acid substrate. In mPGHS-2, Tyr385 was shown to fulfill this role and can be structurally aligned with Tyr374 in the hypothetical PpoA model. Note that, due to the position of the substrate, the position of this residue differs between the two enzymes. While arachidonic acid penetrates the enzyme pocket less deeply, LA lies between Tyr374 and Tyr327 and therefore permits the formation of a hydrogen bond between these residues and leads to a rotation of the tyrosine's side chain. If the substrate penetrates the cavity in PpoA less deeply, Tyr327 could interact with Tyr374 through hydrogen bonds and thus place this tyrosine in a proper position. This role was proposed for the corresponding residue of mPGHS-2 (Tyr348). (F) Residues crucial for P450 activity. In the in silico predicted structure, no basic amino acid was identified at a reasonable distance to the carboxy group of the substrate. Heme cofactor is bound tightly to the apoenzyme by ionic interactions of its propionate groups with Arg944 (no corresponding residue in P. argentatum AOS) and His1004 (Lys424 in P. argentatum AOS). The iron is coordinated by the sulfhydryl group of Cys1006 (Cys426 in P. argentatum AOS). Asn887 is located in the I-helix of the domain and might facilitate $\mathrm{O}-\mathrm{O}$ bond cleavage by a hydrogen network, oriented perpendicular to the hemeiron-peroxide axis. This role was proposed for Asn276 in the homologue enzyme AOS. Note that the two residues do not align structurally. This might indicate an inappropriate model or another role for the Asn887. 
reasonable position in between the heme and the substrate to fulfill its role as mediator of redox chemistry between heme and substrate (Fig. 1D). Nevertheless, while the corresponding distance in mPGHS is $3.6 \AA$, the distance in the hypothetical model is comparably large $(6.9 \AA)$. Interestingly, in the predicted structure the hydroxyl group of Tyr327 is positioned at a closer distance to the fatty acid C8 than that of Tyr374. The homologue position has already been shown to be crucial for catalysis in 7,8-LDS [17]. Based on these observations, a Tyr327Phe and an Arg336Met variant of PpoA were generated and further analyzed.

\section{P450 domain}

While in the predicted N-terminal domain a well defined substrate binding pocket can be identified, the main pocket of the P450 domain of PpoA is predicted to be comparably large. The substrate can be placed in virtually all conformations within this cavity. To get an idea of one likely conformation, we placed the substrate in such a way as it is bound in AOS from guayule (Fig. 1C,E). In this case the carboxy group of the substrate is located next to two positively charged amino acids that could be involved in substrate positioning and may play a role in conferring binding with high affinity: Lys789 and Lys1041 (data not shown). Although, in the modeled state both $\varepsilon$-amino groups seem to be too far apart for strong ionic bonding, enzyme dynamics and a changed folding situation within the entire enzyme could account for this. In contrast to the substrate binding, heme seems to be bound tightly and rigid within the P450-fold. As already seen by sequence alignments, the additional nine-amino-acid insert in the heme binding loop that is typical for Cyp74 enzymes [16] is not present in the heme binding loop of PpoA. Within the structure the heme is stabilized by ionic interactions of positively charged amino acids with the propionate groups of the porphyrin ring. Interaction of one propionate group with an arginine, nine amino acids downstream of the EXXR motif, is typical for virtually all P450 structures [17] and is also seen in the predicted PpoA structure. The role of histidine 1004 in ionic interaction with the other propionate group was already shown by mutational analysis [10]. Changing this charged residue to a hydrophobic one leads to a loss of the heme group without perturbation of the overall structure. Besides the mode of heme binding, the other unique feature of class III P450s in contrast to a typical P450 enzyme can be found in the I-helix [18]. Like in AOS the oxygen binding motif with its conserved threonine is not present in the I-helix of PpoA. Nevertheless the changes in the I-helix are not absolutely identical to those in AOS. The AOS-typical sequence motif that is supposed to be crucial for heme binding and catalytic function is not present in the I-helix of PpoA. Instead, an asparagine that is conserved in all class III P450s and plays a pivotal role in fatty acid rearrangement reactions can be identified in a similar position within the modeled structure (Fig. 1F, Asn887). Note that within this structure the side chain of Asn 887 is shifted $\sim 90^{\circ}$ from the expected position with the amide side chain pointing not perpendicular to the hydroperoxyiron bond. Therefore, an Asn887Val variant was generated and further analyzed.

\section{Enzyme expression and purification}

The three mutants Tyr327Phe, Arg336Met and Asn887Val and wild-type enzyme were expressed and purified following published procedures [10]. Heme occupancy was assured by UV-visible spectroscopy and the ratio of heme absorption to protein absorption $\left(A_{410} / A_{280}\right)$ (Fig. S1A). CD spectroscopy in the far UV range was utilized to verify that the conducted mutations did not lead to a perturbation of the protein fold (Fig. S1B). CD spectra in the visible region were obtained to assure the integrity of the heme cavity and its surrounding active center (Fig. S1C). Protein homogeneity was analyzed by SDS/PAGE (Fig. S2). All variants except Asn887Val showed wild-type behavior for all of these quality criteria. Although this variant was purified to homogeneity and the overall structure, as determined by far-UV CD spectroscopy, was not changed, the mutation of Asn887, located in the I-helix of the P450 domain, to Val caused a reduction of the Soret peak to $\sim 70 \%$ of the wild-type value. This reduced absorption of the Soret peak also affected the $\mathrm{CD}$ signal in the visible range.

\section{Conversion of substrate methyl ester}

To estimate the importance of ionic interactions for affinity and positioning of the substrates, we measured the enzymatic conversion of the methyl ester of the native substrate linoleic acid (LA). The methyl ester of the fatty acid should prevent ionic interactions and, on the other hand, hardly restrict substrate binding for steric reasons. While the enzyme did not convert the methyl ester of LA to any product, the methyl ester of its intermediate DOX product 8-hydroperoxy LA (8-HPODE) was converted to a product (Fig. 2).

The formed product was identified by its retention time and its LC-MS/MS spectrum as the methyl ester of 5,8-dihydoxy LA (5,8-DiHODE) (Fig. S3). 


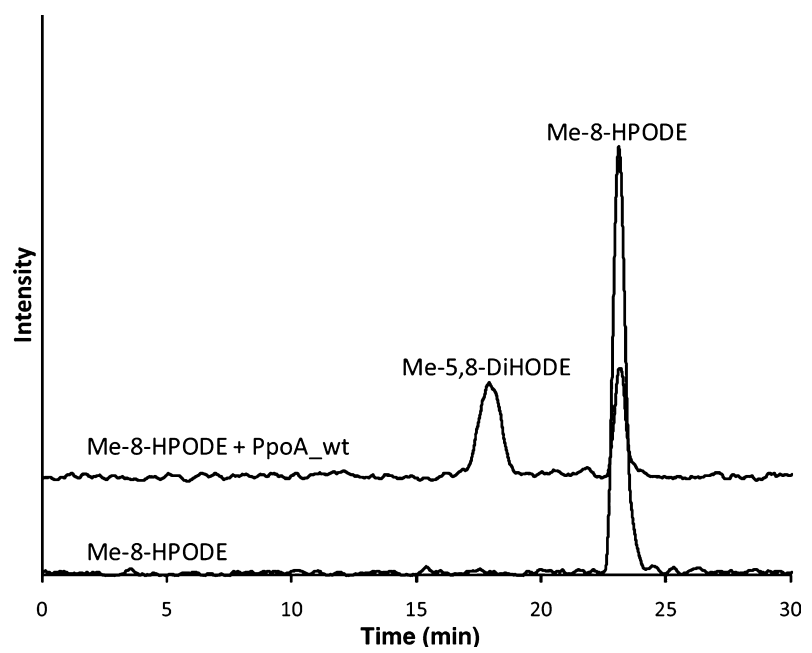

Fig. 2. The methyl ester of 8 -HPODE is converted by PpoA. The lower trace shows the chromatogram of the substrate (methylated8-HPODE) used for conversion with PpoA wild-type. In the upper trace the result of enzymatic conversion is shown. The peak at 17 min can be identified as methyl ester of 5,8-DiHODE (Fig. S3).

The fact that the methyl ester of 8-HPODE was converted in significant amounts and no byproducts were formed points out that ionic interactions neither confer high affinity in substrate binding nor determine substrate positioning in the P450 domain of PpoA.

In contrast to this result for the P450 domain, either ionic interactions in the DOX domain prevent conversion of LA methyl ester or the substrate channel is constrained not to tolerate even a small increase of substrate size. Interestingly, the Arg336Met mutation did not produce an enzyme that was able to convert LA methyl ester.

\section{Determination of kinetic parameters}

To continuously assess the effect of point mutations on substrate binding and conversion, the depletion of dissolved oxygen was monitored. Mutating Arg336 in the N-terminal DOX domain, putatively involved in ionic interaction with the carboxy group of the substrate, to an uncharged methionine reduces the substrate affinity of the enzyme, as reflected by an approximately three-fold increase in $k_{\mathrm{m}}$ (Fig. 3 and Table 1). The fact that the observed $k_{\text {cat }}$ value for this variant was lower than that of the wild-type might be explained by the formation of substrate micelles. Thus, the $k_{\mathrm{m}}$ for this variant might be even higher than the one measured here. Mutation of Tyr327 leads to a drastic reduction of the activity, as illustrated by an

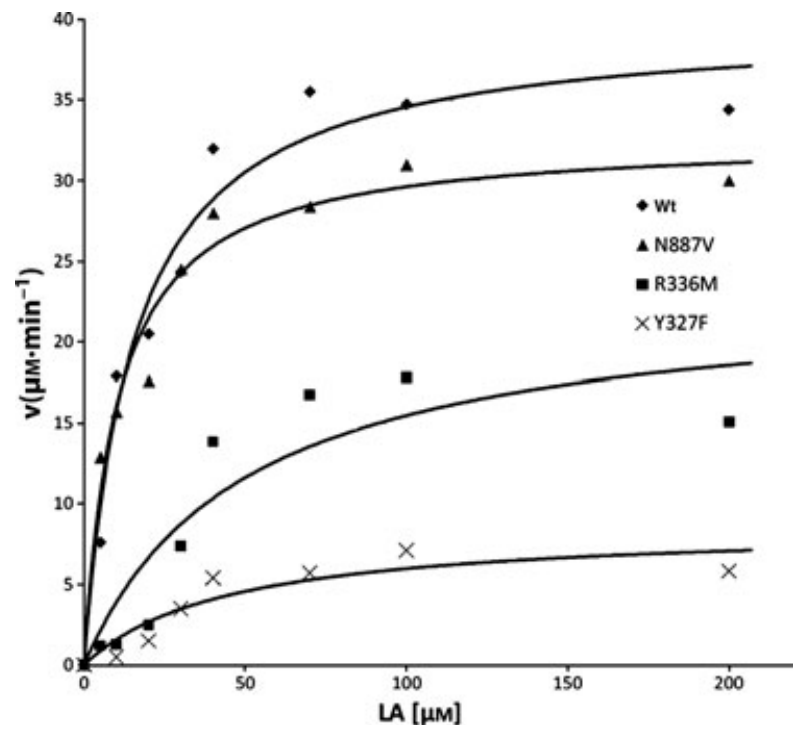

Fig. 3. Kinetics of $L A$ conversion by $P p o A$ variants show the involvement of Arg336 and Tyr327 in substrate binding and dioxygenation.

Table 1. Effects of mutations on macroscopic kinetic constants.

\begin{tabular}{lll}
\hline Mutant & $k_{\mathrm{m}}(\mu \mathrm{M})$ & $k_{\mathrm{cat}}\left(\mathrm{min}^{-1}\right)$ \\
\hline Wild-type & 15.3 & 399 \\
Arg336Met & 49.5 & 231 \\
Tyr327Phe & 44.9 & 87 \\
Asn887Val & 10.5 & 327 \\
\hline
\end{tabular}

almost 14-fold drop in $k_{\mathrm{cat}} / k_{\mathrm{m}}$. Note that for both variants the observed hyperbolic kinetics are changed to a sigmoid shape and therefore description of the data by Michaelis-Menten parameters have to be treated with care. The mutation of Asn887, located in the P450 domain, had only a minor influence on oxygen consumption.

\section{End-product quantification and identification via LC-MS/MS}

To estimate the influence of the respective mutations on the overall reaction, we measured the formation of 5,8-DiHODE, 8-H(P)ODE and 10-hydoxy LA (10-HODE) under substrate (i.e. LA) saturation and analyzed the products formed after $1 \mathrm{~min}$. As expected, the effects observed in the measurement of oxygen depletion are also reflected in the amount of end-product formed. In incubations of PpoA-Arg336Met with LA end-product formation (i.e. formation of 5,8-DiHODE) was roughly reduced by about $70 \%$ while for 
PpoA-Tyr327Phe this value is decreased to $\sim 15 \%$ of the wild-type value. Although the decrease in the activity of the rearrangement reaction, caused by mutating Asn887 to valine, was not reflected significantly in the kinetics obtained by measuring oxygen depletion, we could observe a drastic effect on end-product formation. The amount of 8-HPODE rearranged after $1 \mathrm{~min}$ to 5,8-DiHODE was only approximately one-third of the amount in the wild-type enzyme. The mutations not only affected the quantity of formed products but also influenced the amount of byproducts of the enzymatic reaction (Fig. 4). Not surprisingly, for the mutant Asn887Val, which had a nearly unaffected activity of the DOX domain but was impaired in the rearrangement of the intermediate, 8-HPODE accumulated drastically. More intriguingly, the amount of 8-HODE was significantly increased in both mutants of the DOX domain.

\section{EPR spectroscopy}

Since the mutation of Asn887 caused an intensity decrease of the Soret peak, we investigated whether this is caused by a change in the P450-fold leading to a lower heme occupancy or whether this is merely caused by a change in the ligation sphere of the P450heme that is macroscopically reflected by a changed extinction coefficient. We therefore obtained EPR spectra, in which the spin state of the iron can be differentiated between $\mathrm{Fe}($ III) $S=5 / 2$ high spin heme (DOX domain) and $\mathrm{Fe}(\mathrm{III}) S=1 / 2$ low spin heme (P450 domain) [13]. The obtained spectra of Asn887Val (Figs 5 and S4) showed, indeed, a changed signal ratio of low spin to high spin heme compared with

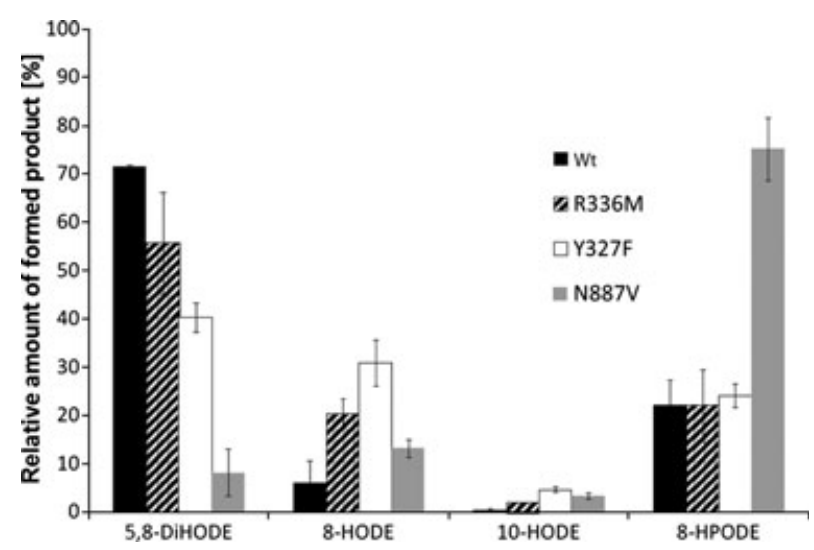

Fig. 4. Relative product formation of PpoA variants. Measured was the product formed after reaction for $1 \mathrm{~min}$ under substrate saturation. Note that mutation of Asn887 affected the rate of 8-HPODE rearrangement and led to an accumulation of this intermediate.

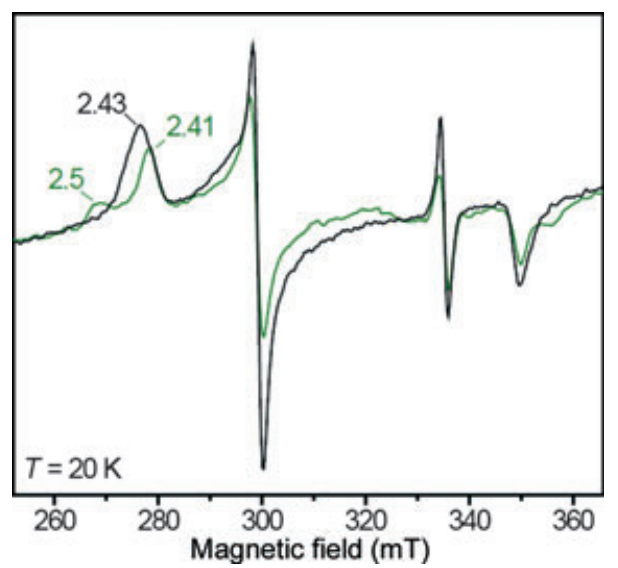

Fig. 5. EPR spectra $(9 \mathrm{GHz})$ of the low spin species of $75 \mu \mathrm{M}$ PpoA and $75 \mu \mathrm{M}$ N887V. The spectra were recorded in HEPES buffer $\mathrm{pH}$ 7.4 at $20 \mathrm{~K}$, microwave power $0.05 \mathrm{~mW}$, modulation amplitude $7 \mathrm{G}, 100 \mathrm{kHz}$ modulation frequency and 10 scans. $g$ values are labeled. Note that the signal at $335 \mathrm{mT}$ arises from overlap of the $g_{z}$ high spin resonance and a minor radical signal.

wild-type PpoA with the double integrated line intensity of low spin Asn887Val reduced by $\sim 40 \%$. We have previously shown that the low spin resonances of PpoA are consistent with thiolate ligation to the heme iron [13]. The principal EPR $g$ values of Asn887Val at $g_{1}=2.41, g_{2}=2.25$ and $g_{3}=1.91$ are consistent with thiolate ligation (see Fig. 5). They show a shift of the $g_{1}$ resonance compared with the native enzyme $\left(g_{1}=2.43\right)$. Some minor variations in the linewidth of the outer $g_{1}$ and $g_{3}$ resonances are noted. The $g$ values are very sensitive to the nature of the heme axial ligands and to ligand orientations. The observed $g$ value shift and linewidth variations here indicate slight changes in the flexibility and strength of the axial linkages to the heme iron in the low spin domain. We also observe some heterogeneity of the low spin resonances with a minor species with $g_{1}=2.5$. This changed heme ligation sphere might also explain the lower heme occupancy of the P450 domain.

To address the question of Tyr327 affecting the hydrogen bonding network about Tyr374 we manually mixed (8R)-HPODE with Tyr327Phe similar to our previous mixing experiments with PpoA that generated a tyrosyl radical [13]. We observed generation of a radical signal at $g=2$ with altered linewidths compared with that observed with PpoA (Fig. S5). The radical intensity was reduced by $15 \%$ compared with the wildtype enzyme. High field $(95 \mathrm{GHz})$ EPR was performed in order to identify the radical species. We observed a species with $g$ anisotropy consistent with that of a tyrosyl radical (Fig. S5 and [13]). It was not possible 
to simulate the spectrum with one species, which suggested the presence of a second radical species. Further spectroscopic work at higher frequencies would be needed to identify the second radical. The identified tyrosyl appears to have a narrower $\beta$-proton splitting $(\sim 16 \mathrm{G})$ compared with wild-type $(\sim 20 \mathrm{G})$, indicative of a change in dihedral angle between the $\beta$-protons and the phenoxyl ring.

\section{Discussion}

Modeling of PpoA was based on the idea that the DOX as well as the P450 domain might be modeled adequately due to high structural conservation within the respective enzyme family. Therefore, domain borders were defined after amino acid residue 620 and both sequences were submitted to the I-TASSER algorithm for structure prediction. This server combines homology modeling, protein threading and de novo prediction algorithms $[19,20]$. Due to the high conservation of the cysteine binding loop of the heme within P450s [21], this cofactor can be placed within the structure with high fidelity. For the DOX domain previous electron nuclear double resonance measurements allowed the unequivocal identification of a histidine as the fifth heme ligand [13]. Moreover, it was shown that the heme binding cavity within the myeloperoxidase family is conserved even for subfamilies showing distinct properties [22]. Therefore the position of this histidine can be identified by alignment with the related enzymes PGHS-1 and PGHS-2. Using this constraint, the heme of the N-terminal domain can also be positioned with high fidelity.

\section{DOX domain}

The hypothetical model of this domain was able to place various known determinants of dioxygenation in a reasonable spatial arrangement. The heme is positioned between two histidines: His 377 on the proximal site of the heme plane seems to coordinate the iron as fifth ligand, while His202 is located on the distal site of the heme. The essential role of these amino acids for activity of the related 7,8-LDS has already been proven by site-directed mutagenesis elsewhere [23]. In the same study Tyr374 and Tyr327 (PpoA numbering) were shown to be involved in substrate oxidation. Finally, Val328 seems to play a certain role in determination of regio specificity of the oxygen insertion [24]. While this role was only weakly pronounced in PpoA, the enzyme PpoC, which catalyzes formation of a $10 R$-hydroperoxy fatty acid, was shown to be quite sensitive for a mutation of the corresponding residue [25]. Shielding C3 and $\mathrm{C} 4$ of the fatty acid substrate, Val328 is positioned in a situation identical to its position in PGHS [24]. Additionally to this known determinant, an arginine (Arg336) could be identified that seems to be engaged in ionic interactions with the substrate's carboxyl group and therefore confers substrate affinity and places the substrate in a preferred orientation (Fig. 1D). Not surprisingly, mutation of this charged residue did not completely abolish the DOX activity (Table 2). Thus a significant contribution to substrate affinity seems to be governed by hydrophobic interactions of the fatty acid tail with hydrophobic residues lining the substrate binding pocket (i.e. Leu139, Ala197, Ile200, Ile323, Leu324, Ile557, Ala561, Leu564). A future study utilizing isothermal titration calorimetry may help to decipher the entropic and enthalpic contributions to substrate binding. Since also mutation of this arginine to the uncharged methionine did not result in conversion of LA methyl ester, we deduce that either the substrate channel is indeed very narrow and does not tolerate any increase in substrate size or that the predicted substrate binding pocket represents only the final end of a deeper pocket ranging from the protein surface to the active center of the DOX domain, in which the substrate finally is held in the right position by interaction with Arg336. Actually, this longer substrate channel might be formed by the interface of the two domains and therefore is not predictable by the approach of modeling the single domains.

In addition, we suggest an essential role of Tyr327 in catalysis. The corresponding residue in PGHS-2 is known to form a hydrogen bonding network with the tyrosine that forms the essential radical, which finally mediates hydrogen abstraction from the fatty acid [26]. Here it was shown that the formed hydrogen bonds are of importance to place this radical in a proper orientation within the substrate binding pocket. Within the minimized structural model, we could observe an intercalation of the fatty acid between the corresponding PpoA tyrosines (Fig. S6A). Since this argued against a hydrogen bond in PpoA, we conducted sitedirected mutagenesis to elucidate the role of Tyr327. The respective variant showed kinetically impaired substrate conversion, suggesting an essential role of this residue. Interestingly, the respective EPR spectrum of the tyrosyl radical produced by Tyr327Phe on addition of substrate showed not only a reduced intensity of radical formed but also an altered line shape compared with the wild-type spectrum. This indicates an altered geometry compared with the radical observed in the wild-type and suggests that Tyr327 in PpoA may have a similar role to Tyr348 in PGHS-2. This result exemplifies that the substrate position within the DOX domain may not be reflected in an entirely 
correct way by the modeled structure and a slightly changed position of the substrate which allows the formation of a hydrogen bond between Tyr327 and Tyr374 (Fig. S6B) might be more realistic, especially since this would allow the position of Tyr374 at a more reasonable distance to the substrate's C8. Moreover, we could show that both variants (PpoAArg336Met and PpoA-Tyr327Phe) show a change in the shape of the observed kinetics. This is most likely caused by an impaired formation of activating peroxide and might reflect the complex non-Michalis-Menten kinetics with steps of enzyme activation and inactivation that are a central part of the dioxygenation mechanism [12]. However, it has to be considered that micelles are formed in a concentration higher than $\sim 100 \mu \mathrm{M}$. Thus, the evaluation of the kinetic data is complicated and especially the kinetic constants for both DOX domain variants (i.e. Arg336Met and Tyr327Phe) might be misinterpreted.

\section{P450 domain}

Not surprisingly, the overall structure of the modeled P450 domain shows the conserved structure of all P450s. The heme is tightly bound by coordination of the iron to the sulfhydryl of Cys1006 and interactions of the protoporphyrin IX's propionate groups with Arg944 and His1004. While the position of the cofactor is well defined by these determinants, the hydroperoxide substrate could be placed in various confirmations within the predicted structure. Moreover, specific conversion of 8-HPODE methyl ester showed that the position of the substrate is not determined by ionic interactions and that the binding pocket of this domain tolerates an increase in substrate size. Also in line with a flexible substrate positioning in Cyp74 and related enzymes is the fact that the substrate in two solved structures of AOS (3DBM [18] and $2 \mathrm{RCH}$ [27]) is oriented in a different way (Fig. S7). Although the overall structures match very well (rmsd $=0.822 \AA$ over 436 matched Ca-atoms), the co-crystallized substrate analogues are placed with a changed head-to-tail orientation within the substrate cavity. Nevertheless, the distance between the peroxide and the iron might be too large in guayule AOS (3DBM) to reflect a productive mode of substrate binding. The observed conversion of hydroperoxy fatty acid methyl ester without change in reaction specificity by a class III P450 and the flexible orientation of 13-HODE within AOS exemplify the obviously weak constrained substrate positioning within this class of P450s. For the above mentioned reasons, it is not possible to predict the ultimate catalytic productive posi- tion of the substrate within the P450 domain of PpoA. Knowing that the proposed formation of diols from a hydroperoxy precursor does not involve severe substrate repositioning [28], different substrate orientations with $\mathrm{C} 5$ being in closer proximity to the heme might be more realistic than the one proposed here. But, since the native substrate orientation is not defined by ionic interactions, experimental probing of a certain confirmation out of the multitude of possible ones is not feasible. A crystal structure of this domain with bound substrate might give an idea about the correct substrate position. Independent from the orientation of the remaining substrate atoms, the position of the peroxide linearly above the iron on the distal side of the heme is crucial for catalysis. Based on this orientation, we could identify Asn887 and EPR analysis of a respective variant showed perturbation of the low spin heme environment. Thus, the crucial presence of Asn887 might indicate a hydrogen bonding network connecting the amide and the iron. This observation is in agreement with the role of asparagine residues in other class III P450s [16,29,30]. Interestingly, the position of the asparagine identified here deviates by one residue from the corresponding position in homologue class III P450s. Nevertheless, since we observed the possibility of a changed hydrogen bonding network with the iron, the amide side chain has to be in the heme proximity and the observed position might be an artifact, caused by the modeling process trapped in a minimum of the energy function or a false prediction of the starting residue of the helix. Thus, this amide side chain most likely forms a hydrogen network to promote the fission of the peroxide $\mathrm{O}-\mathrm{O}$ bond (Fig. 6A). Although all other studied class III P450 enzymes have been reported to catalyze their respective rearrangement reactions by homolytic cleavage of the peroxide $\mathrm{O}-\mathrm{O}$ bond $[16,29]$, the rearrangement reaction catalyzed by PpoA-like enzymes and leading to the formation of dihydroxy fatty acids is most likely caused by heterolytic cleavage of this bond [28]. This has some implications for the mechanism of this enzymatic conversion. While typical class III P450s shortcut the commonly accepted reaction cycle and yield compound II directly, the reaction cycle in the P450 domain of Ppo enzymes includes compound I as an additional heme intermediate (Fig. 6B). The rearrangement is initiated by heterolytic $\mathrm{O}-\mathrm{O}$ bond cleavage, yielding 8-HODE and compound I. Subsequently, the commonly accepted $\mathrm{P} 450$ reaction cycle might proceed including hydrogen abstraction from C5 of the substrate and oxygen rebound to the formed carbon centered radical. The main determinant for hetero- versus homolytic $\mathrm{O}-\mathrm{O}$ bond fission might be the large 
A

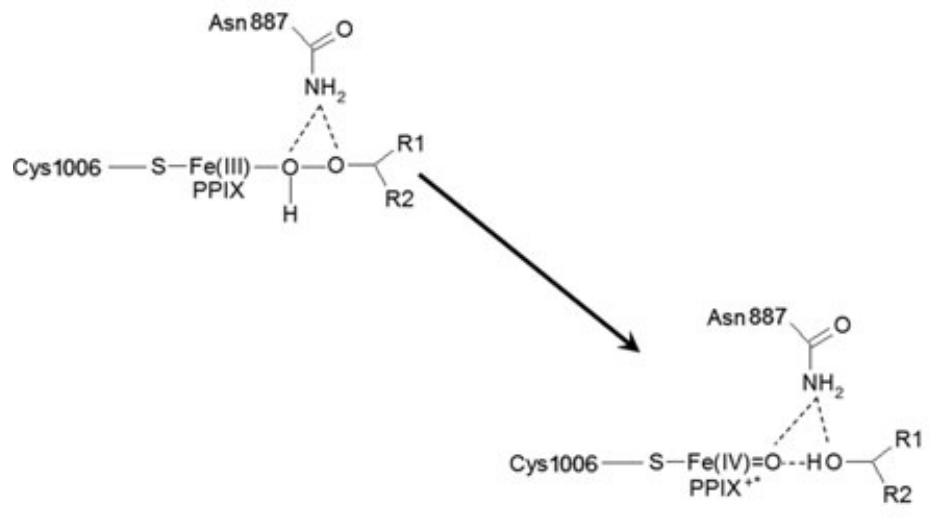

B

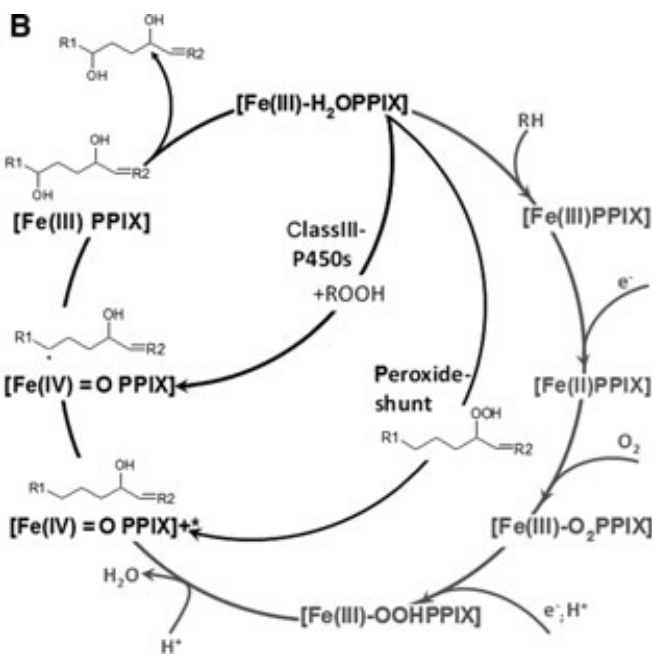

Fig. 6. Proposed mechanism of fatty acid peroxide rearrangement within the P450 domain of PpoA. (A) The proposed role of Asn887 as a determinant to facilitate peroxide bond cleavage. The mechanism is adapted from Lee et al. [27]. (B) Reaction cycle of hydroperoxy fatty acid rearrangement. In typical class III P450s, the classical P450 reaction cycle is shortcut and heme compound II is directly yielded after homolytic cleavage of the substrate peroxide. In contrast, the reaction catalyzed by PpoA most likely follows the peroxide shunt of P450s. Note that homolytic cleavage of the substrate's O-O bond according to the reaction catalyzed by typical class III P450s would yield an allylic alkoxyl radical. The reaction cycle was adapted and modified according to Brash and Denisov et al. [16,34].

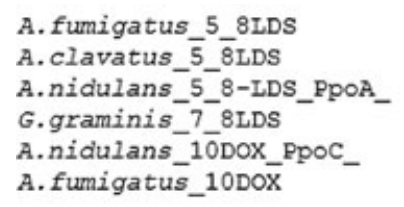

A.fumigatus_5_8IDS A. clavatus $\overline{5} \overline{8}$ LDS A.nidulans_5_8-LDS_PpoA G. graminis 7 - 8 LDS A.nidulans_10 $100 \mathrm{X} \_$PpoC_ A. fumigatus__10DOX
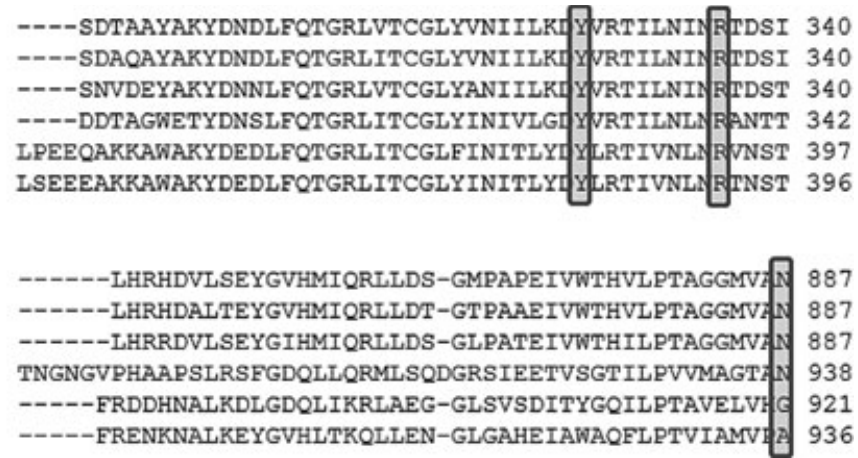

Fig. 7. Sequence alignments of Ppo enzymes show the catalytic relevance of the residues identified in this study. Aspergillus fumigatus_5,8-LDS (UniProt Q4WPX2), Aspergillus clavatus_5,8-LDS (UniProt A1Cl17), A. nidulans_5,8-LDS (PpoA) (UniProt Q6RET3) and G. graminis_7,8-LDS (UniProt $\overline{\text { Q9UUS2) }}$ catalyze formation of 8-HPODE within their N-terminal domain and subsequently rearrange this peroxide to a diol. In contrast, A. nidulans_10DOX (PpoC) (UniProt Q6IYE5) and A. fumigatus_10DOX (UniProt Q4WY82) catalyze the formation of a 10 hydroperoxy adduct and do not rearrange this within their P450 domain. Therefore, the asparagine within the I-helix is not conserved for these enzymes.

substrate binding cavity that allows water to bind and participate in acid catalyzed bond cleavage [31]. Although the substrate size is the same for the rearrangements catalyzed by AOS and PpoA, the main pocket of the enzyme is 1.6-fold larger in the structure modeled here. Even the main pocket of PGIS possesses only $\sim 70 \%$ of the volume of the modeled P450, although the substrate of this enzyme is much bulkier. This larger binding cavity is consistent with a higher accessibility of aqueous solvent in order to facilitate heterolytic bond fission.
Interestingly, sequence alignments of known Ppo enzymes from several ascomycetes indicate that the identified residues are indeed conserved in all sequences, pointing out their relevance for catalysis (Fig. 7).

The reductionist approach to model the domains independently from each other abstracts away one of the most intriguing features of Ppo enzymes: the combination of two well known enzymatic functionalities in one polypeptide chain. Depending on the orientation of the domains to each other and the interaction 
of loops from one domain with the backbone of the other, new constraints will arise that might govern the protein fold. Since this linker region is something unique for Ppo enzymes and no adequate homologue structure is available, these parameters cannot be included in the enzyme model. Moreover, molecular dynamics could not be considered in this study and alternative views on substrate positioning might be possible in the native context of the bifunctional enzyme. In order to improve the modeled structure and include the influence of domain interactions, additional constraints, e.g. derived by small angle X-ray scattering, would be necessary. Nevertheless, the prediction of protein folds is still an error-prone approach and the incompleteness of the hypothetical models presented here is illustrated by several issues. Within the DOX domain the position of LA is inconsistent with a hydrogen bonding network between Tyr374 and Tyr327 and the distance between Tyr374 and C8 of the substrate is approximately two-fold larger than the corresponding distance in mPGHS-2. This might be explained by molecular dynamics or it might be seen as an indication of an inappropriate substrate placement within the model. In the P450 domain the side chain of Asn 887 is shifted by $90^{\circ}$ from the expected position perpendicular to the peroxide-Fe axis. Taken together the results illustrate nicely that a model can be well suited to identify putative determinants but interpretation of their respective role has to be treated with caution and validation by other approaches is necessary. Although the unique linker cannot be predicted by the approach here and the model does not reflect an entirely realistic situation on an atomic level, we were able to determine the overall-fold of the single domains and could show that structure and mechanism of oxylipin-forming enzymes may be conserved throughout all kingdoms of life. Based on the predicted structures, we could identify new determinants that are involved in the placement of the substrate, correct orientation of the oxidizing tyrosyl radical and hydroperoxy fatty acid rearrangement.

\section{Materials and methods}

\section{In silico prediction of substrate position within the active sites}

The amino acid sequence of PpoA (UniProt-ID Q6RET3) was submitted in two independent strings to the I-TASSER algorithm (http://zhanglab.ccmb.med.umich.edu/I-TASSER/) [22,23]. Amino acids 1-620 were considered to fold the $\mathrm{N}$-terminal DOX domain of the enzyme, while the sequence from amino acid 620 to 1081 was submitted to yield a structural model of the C-terminal P450 functionality of PpoA. No further templates and restraints were defined to guide the threading process. The resulting apodomains were loaded into the molecular modeling program USCF CHIMERA 1.5.2 [32] and superimposed with the structure of mPGHS2 (PDB 3HS5, chain B) in case of the DOX domain and the structure of CYP74A2 (PDB 3DBM) in case of the P450 domain. For both domains, initial placement of the heme cofactor was determined by its position within the native structures of these homologous enzymes. Substrates of PpoA (LA and 8-HPODE) were manually placed to fit the position of the substrates in the crystal structures of the homologous enzymes. After removal of the superimposed enzymes, the modeled holo domains were refined within the Amber forcefield, implemented in the USCF CHIMERA package. Pocket volumes were calculated with the CastP algorithm [33].

\section{Materials}

Oligonucleotides were purchased from Sigma-Aldrich (Steinheim, Germany). Pfu DNA- Polymerase and DpnI were from MBI-Fermentas (St Leon-Rot, Germany) and all other chemicals, unless stated otherwise, were obtained from Carl Roth GmbH (Karlsruhe, Germany) and were of the highest purity available.

\section{Site-directed mutagenesis}

Site-specific nucleotide exchanges were conducted on the previously described expression plasmid [10] by utilization of oligonucleotides with specific mismatches at the desired position. PCR amplification was performed by $P f u$-Polymerase according to the manufacturer's instructions. The sequence of oligonucleotides is given in Table 2. After 25 cycles of PCR, template DNA was digested with $D p n I$ and the mutated plasmid was transformed to XL1- Blue (Invitrogen, Darmstadt, Germany). Insertion of site-specific mutations was confirmed by sequencing of the entire open reading frame.

Table 2. Oligonucleotides, utilized for site-directed mutagenesis: mismatches are depicted in capitals.

\begin{tabular}{ll}
\hline $\begin{array}{l}\text { Oligonucleotide } \\
\text { name }\end{array}$ & Sequence \\
\hline R336M_sense & gaatataaacATgacagatagcacctggagtttgg \\
R336M_antisense & gctatctgtcATgtttatattcaaatcgttcgg \\
Y327F_sense & $\begin{array}{l}\text { aagattTtgtccgaacgattttgaatataaaccgg } \\
\text { cggacaAaatcttttaggataatatttgcg }\end{array}$ \\
Y327F_antisense & aatggtggcaGTccaagcacaaTtgtttcgc \\
N887V_antisense & gcgaaaacattgtgcttggactgccaccatt \\
\hline
\end{tabular}




\section{Enzyme expression and purification}

Expression and purification of wild-type enzyme and all variants followed the previously described protocols [10].

\section{Conversion of methylated substrate}

$10 \mu \mathrm{L}$ of $100 \mu \mathrm{Ci} \cdot \mathrm{mL}^{-1} 1{ }^{14} \mathrm{C}-\mathrm{LA}$ (Perkin Elmer, Waltham, MA, USA) and $5 \mu \mathrm{L}$ of $500 \mathrm{~g} \cdot \mathrm{L}^{-1}$ peroxide-free LA (Cayman, Ann Arbor, MI, USA) were mixed in $400 \mu \mathrm{L}$ methanol and converted to methylated LA by addition of $10 \mu \mathrm{L} 2 \mathrm{M}$ trimethylsilyl-diazomethan (Sigma-Aldrich) in hexane. The reaction was accomplished on a shaker at room temperature for $30 \mathrm{~min}$. After evaporation of the solvent under a gentle nitrogen stream, LA methyl ester was separated from the remaining free fatty acid by RP-HPLC. HPLC conditions were chosen identical those of Brodhun et al. [10]. ${ }^{14} \mathrm{C}$-labeled 8 -HPODE was produced by conversion of $10 \mu \mathrm{L}$ of $100 \mu \mathrm{Ci} \cdot \mathrm{mL}^{-1} 1-{ }^{14} \mathrm{C}$-LA and $5 \mu \mathrm{L}$ of $500 \mathrm{~g} \cdot \mathrm{L}^{-1}$ peroxide-free LA by PpoAHis1004Ala. The fatty acid peroxide product was isolated by RP-HPLC and subsequently the corresponding methyl ester was furnished by reaction with trimethylsilyl-diazomethan. The methylated 8-HPODE was again isolated by RP-HPLC. These substrate methyl esters were finally converted with $100 \mathrm{nM}$ of the respective enzyme in $20 \mathrm{~mm}$ HEPES, pH 7.4, for $30 \mathrm{~min}$ at room temperature. After extraction with diethyl ether, the conversion products were analyzed by RP-HPLC coupled to a Raytest scintillation counter.

\section{Measurement of kinetics and product identification via LC-MS/MS}

Kinetics were measured on a Clark-type electrode and endproduct formation as well as product pattern was determined by LC-MS/MS. Both methods have been described previously [12].

\section{EPR spectroscopy}

Continuous-wave (CW) EPR spectra were recorded at $9 \mathrm{GHz}$ using a Bruker ELEXSYS E500 with a Bruker SuperX CW bridge. The spectrometer was equipped with a super high sensitivity probe head and a liquid helium cryostat (Oxford Instruments, Abingdon, UK). Manually mixed EPR samples were prepared and spin quantitation was performed as previously described [13]. High frequency EPR spectroscopy at $94 \mathrm{GHz}$ was recorded using a Bruker Elexsys 680 spectrometer (Power Upgrade 2). The magnetic field was calibrated as previously described [13]. Samples were mixed into $0.9 \mathrm{~mm}$ outer diameter, $0.5 \mathrm{~mm}$ inner diameter quartz capillaries. All EPR conditions are stated in the figure captions.

\section{Acknowledgements}

We are grateful for financial support from the German Research Foundation (International Research Training Group 1422, Metal Sites in Biomolecules: Structures, Regulation and Mechanisms). In addition C.K. was supported by the Biomolecules program of the Göttingen Graduate School of Neurosciences and Molecular Biology (GGNB). The help of Dr Danilo Meyer with $\mathrm{CD}$ spectroscopy is greatly acknowledged. We are grateful to Benjamin Göde for expert technical assistance. Molecular modeling and imaging was done using the UCSF Chimera package from the Resource for Biocomputing, Visualization, and Informatics at the University of California, San Francisco (supported by NIH P41 RR001081).

\section{References}

1 Andreou A, Brodhun F \& Feussner I (2009) Biosynthesis of oxylipins in non-mammals. Prog Lipid Res 48, 148-170.

2 Champe SP \& el-Zayat AA (1989) Isolation of a sexual sporulation hormone from Aspergillus nidulans.

J Bacteriol 171, 3982-3988.

3 Tsitsigiannis DI \& Keller NP (2006) Oxylipins act as determinants of natural product biosynthesis and seed colonization in Aspergillus nidulans. Mol Microbiol 59, 882-892.

4 Tsitsigiannis DI \& Keller NP (2007) Oxylipins as developmental and host-fungal communication signals.

Trends Microbiol 15, 109-118.

5 Brodhun F \& Feussner I (2011) Oxylipins in fungi. FEBS J 278, 1047-1063.

6 Christensen SA \& Kolomiets MV (2011) The lipid language of plant-fungal interactions. Fungal Genet Biol 48, 4-14.

7 Tsitsigiannis DI, Bok JW, Andes D, Nielsen KF, Frisvad JC \& Keller NP (2005) Aspergillus cyclooxygenaselike enzymes are associated with prostaglandin production and virulence. Infect Immun 73, 4548-4559.

8 Tsitsigiannis DI, Kowieski TM, Zarnowski R \& Keller NP (2005) Three putative oxylipin biosynthetic genes integrate sexual and asexual development in Aspergillus nidulans. Microbiology 151, 1809-1821.

9 Brodhagen M, Tsitsigiannis DI, Hornung E, Goebel C, Feussner I \& Keller NP (2008) Reciprocal oxylipinmediated cross-talk in the Aspergillus-seed pathosystem. Mol Microbiol 67, 378-391.

10 Brodhun F, Gobel C, Hornung E \& Feussner I (2009) Identification of PpoA from Aspergillus nidulans as a fusion protein of a fatty acid heme dioxygenase/peroxidase and a cytochrome P450. J Biol Chem 284, 1179211805 . 
11 Brodowsky ID, Hamberg M \& Oliw EH (1992) A linoleic acid $(8 R)$-dioxygenase and hydroperoxide isomerase of the fungus Gaeumannomyces graminis. $J$ Biol Chem 267, 14738-14745.

12 Nadler A, Koch C, Brodhun F, Wehland J-D, Tittmann K, Feussner I \& Diederichsen U (2011) Influence of substrate dideuteration on the reaction of the bifunctional heme enzyme Psi factor producing oxygenase A (PpoA). ChemBioChem 12, 728-737.

13 Fielding AJ, Brodhun F, Koch C, Pievo R, Denysenkov V, Feussner I \& Bennati M (2011) Multifrequency electron paramagnetic resonance characterization of PpoA, a CYP450 fusion protein that catalyzes fatty acid dioxygenation. $\mathrm{J}$ Am Chem Soc 133, 9052-9062.

14 Garscha U, Jerneren F, Chung D, Keller NP, Hamberg M \& Oliw EH (2007) Identification of dioxygenases required for Aspergillus development: studies of products, stereochemistry, and the reaction mechanism. $J$ Biol Chem 282, 34707-34718.

15 Werck-Reichhart D \& Feyereisen R (2000) Cytochromes P450: a success story. Genome Biol 1, reviews3003.3001-reviews3003.3009.

16 Brash AR (2009) Mechanistic aspects of CYP74 allene oxide synthases and related cytochrome P450 enzymes. Phytochemistry 70, 1522-1531.

17 Seifert A \& Pleiss J (2009) Identification of selectivitydetermining residues in cytochrome P450 monooxygenases: a systematic analysis of the substrate recognition site 5. Proteins 74, 1028-1035.

18 Li L, Chang Z, Pan Z, Fu Z-Q \& Wang X (2008) Modes of heme binding and substrate access for cytochrome P450 CYP74A revealed by crystal structures of allene oxide synthase. Proc Natl Acad Sci USA 105, 13883-13888.

19 Zhang Y (2008) I-TASSER server for protein 3D structure prediction. BMC Bioinformatics 9, 40.

20 Roy A, Kucukural A \& Zhang Y (2010) I-TASSER: a unified platform for automated protein structure and function prediction. Nat Protoc 5, 725-738.

21 Sirim D, Widmann M, Wagner F \& Pleiss J (2010) Prediction and analysis of the modular structure of cytochrome P450 monooxygenases. BMC Struct Biol 10, 34.

22 Liu W, Rogge CE, Bambai B, Palmer G, Tsai A-L \& Kulmacz RJ (2004) Characterization of the heme environment in Arabidopsis thaliana fatty acid $\alpha$-dioxygenase-1. J Biol Chem 279, 29805-29815.

23 Garscha U \& Oliw EH (2008) Critical amino acids for the $8(R)$-dioxygenase activity of linoleate diol synthase. A comparison with cyclooxygenases. FEBS Lett 582, 3547-3551.

24 Garscha U \& Oliw EH (2009) Leucine/valine residues direct oxygenation of linoleic acid by $(10 R)$ - and $(8 R)$ dioxygenases: expression and site-directed mutagenesis of $(10 R)$-dioxygenase with epoxyalcohol synthase activity. J Biol Chem 284, 13755-13765.

25 Brodhun F, Schneider S, Göbel C, Hornung E \& Feussner I (2010) PpoC from Aspergillus nidulans is a fusion protein with only one active haem. Biochem $J \mathbf{4 2 5}$, 553-565.

26 Rogge CE, Ho B, Liu W, Kulmacz RJ \& Tsai AL (2006) Role of Tyr348 in Tyr385 radical dynamics and cyclooxygenase inhibitor interactions in prostaglandin $\mathrm{H}$ synthase-2. Biochemistry 45, 523-532.

27 Lee D-S, Nioche P, Hamberg M \& Raman CS (2008) Structural insights into the evolutionary paths of oxylipin biosynthetic enzymes. Nature 455, 363-368.

28 Kupfer R, Liu SY, Allentoff AJ \& Thompson JA (2001) Comparisons of hydroperoxide isomerase and monooxygenase activities of cytochrome P450 for conversions of allylic hydroperoxides and alcohols to epoxyalcohols and diols: probing substrate reorientation in the active site. Biochemistry $\mathbf{4 0}$, 11490-11501.

29 Hecker M \& Ullrich V (1989) On the mechanism of prostacyclin and thromboxane A2 biosynthesis. $J$ Biol Chem 264, 141-150.

30 Yeh HC, Hsu PY, Wang JS, Tsai AL \& Wang LH (2005) Characterization of heme environment and mechanism of peroxide bond cleavage in human prostacyclin synthase. Biochim Biophys Acta 1738, 121-132.

31 Correia MA, Yao K, Allentoff AJ, Wrighton SA \& Thompson JA (1995) Interactions of peroxyquinols with cytochromes P450 2B1, 3A1, and 3A5: influence of the apoprotein on heterolytic versus homolytic O-O bond cleavage. Arch Biochem Biophys 317, 471-478.

32 Pettersen EF, Goddard TD, Huang CC, Couch GS, Greenblatt DM, Meng EC \& Ferrin TE (2004) UCSF Chimera - a visualization system for exploratory research and analysis. J Comput Chem 25, 1605-1612.

33 Dundas J, Ouyang Z, Tseng J, Binkowski A, Turpaz Y \& Liang J (2006) CASTp: computed atlas of surface topography of proteins with structural and topographical mapping of functionally annotated residues. Nucleic Acids Res 34, W116-W118.

34 Denisov IG, Makris TM, Sligar SG \& Schlichting I (2005) Structure and chemistry of cytochrome P450. Chem Rev 105, 2253-2278.

\section{Supporting information}

The following supplementary material is available:

Fig. S1. Absorbance and CD spectra of PpoA_wt and various mutants. 
Fig. S2. SDS/PAGE of purified PpoA wild type and variants.

Fig. S3. Identification of the peak eluting at $16.7 \mathrm{~min}$ as methyl ester of 5,8-DiHODE.

Fig. S4. 9-GHz EPR spectra of PpoA $(75 \mu \mathrm{M})$ and N887V $(75 \mu \mathrm{M})$.

Fig. S5. (A) 9-GHz CW spectrum at $70 \mathrm{~K}$ of the radical formed after reaction of either PpoA $(75 \mu \mathrm{M})$ (black) or Y327F (blue) with 160 -fold excess $(8 R)$ HPODE. (B) 95-GHz Echo-detected spectrum (1-st derivative) of the radical formed after reaction of either PpoA (75 mM) (black) or Y327F (blue) with 160 -fold excess $(8 R)$-HPODE.
Fig. S6. Superimposition of the modeled DOX domain of PpoA and oPGHS-1.

Fig. S7. Superimposition of the structures of Arabidopsis thalania and Parthenium argentatum AOS.

This supplementary material can be found in the online version of this article.

Please note: As a service to our authors and readers, this journal provides supporting information supplied by the authors. Such materials are peer-reviewed and may be re-organized for online delivery, but are not copy-edited or typeset. Technical support issues arising from supporting information (other than missing files) should be addressed to the authors. 